\title{
Numerical algorithms for the time-space tempered fractional Fokker-Planck equation
}

\author{
Xiaorui Sun, Fengqun Zhao* (D) and Shuiping Chen
}

"Correspondence: zhaofa@xaut.edu.cn

School of Sciences, Xi'an University of Technology, Xi'an, 710054, China

\begin{abstract}
This paper aims to provide the high order numerical schemes for the time-space tempered fractional Fokker-Planck equation in a finite domain. The high order difference operators, called the tempered and weighted and shifted Lubich difference operators, are used to approximate the time tempered fractional derivative. The spatial operators are discretized by the central difference methods. We apply the central difference methods to the spatial operators and obtain that the numerical schemes are convergent with orders $O\left(\tau^{q}+h^{2}\right)(q=1,2,3,4,5)$. The stability and convergence of the first order numerical scheme are rigorously analyzed. And the effectiveness of the presented schemes is testified with several numerical experiments. Additionally, some physical properties of this diffusion system are simulated.
\end{abstract}

Keywords: tempered fractional Fokker-Planck equation; weighted and shifted Lubich difference operators; fractional central difference operator; stability; convergence

\section{Introduction}

In recent decades, fractional partial differential equations have become a powerful tool to model the particle transport in anomalous diffusion in various fields. Effectively solving them naturally becomes an urgent topic of researchers. Luckily, some important achievements have been made for the fractional differential equations [1-10]. Recently, the tempered anomalous diffusion equations [11-13] have drawn the wide interests of the researchers. It is closer to reality in the sense of the finite life span or bounded physical space of the diffusion particles. The detailed introductions about the definitions and properties of the tempered fractional calculus can be seen in [14-17] and the references therein. As the generalization of fractional calculus, tempered fractional calculus does not simply have the properties of the fractional calculus, but can describe some of the other complex dynamics [18]. Tempered fractional derivatives and the corresponding tempered fractional differential equations have played a key role in physics [19], ground water hydrology [20], finance [21], poroelasticity [22], and so on.

In the continuous time random walk (CTRW) model, the corresponding time tempered fractional Fokker-Planck equation is derived [18, 23], where the jump length for Brownian particles is a constant. In practical application, however, the Brownian particles contain arbitrary distance and direction. Moreover, if the jump length probability $\eta(x)$ follows the

(c) The Author(s) 2017. This article is distributed under the terms of the Creative Commons Attribution 4.0 International License (http://creativecommons.org/licenses/by/4.0/), which permits unrestricted use, distribution, and reproduction in any medium, provided you give appropriate credit to the original author(s) and the source, provide a link to the Creative Commons license, and indicate if changes were made. 
Lévy distribution $[24,25]$, i.e., $\eta(x) \simeq|x|^{-\beta-1}(0<\beta \leq 2)$, then the following time-space tempered fractional Fokker-Planck equation can well describe the probability density of particles $[13,18,26]$ :

$$
\frac{\partial u(x, t)}{\partial t}={ }_{0} D_{t}^{1-\alpha, \lambda}\left[-\frac{\partial}{\partial x} F(x)+\frac{\partial^{\beta}}{\partial|x|^{\beta}}\right] u(x, t)-\lambda u(x, t)
$$

where the tempering parameter $\lambda \geq 0$, the function $F(x)$ is the external force, $u(x, t)$ representing the probability density, ${ }_{0} D_{t}^{\alpha, \lambda}$ denotes the left Riemann-Liouville tempered fractional derivative of order $\alpha \in(0,1)$, which is defined as [16]

$$
{ }_{0} D_{t}^{\alpha, \lambda} u(t)=e^{-\lambda t}{ }_{0} D_{t}^{\alpha}\left(e^{\lambda t} u(t)\right)=\frac{e^{-\lambda t}}{\Gamma(1-\alpha)} \frac{d}{d t} \int_{0}^{t}(t-\tau)^{-\alpha} e^{\lambda \tau} u(\tau) d \tau,
$$

where $\partial^{\beta} / \partial|x|^{\beta}$ is the Riesz fractional derivative of order $\beta$ defined by [27]

$$
\frac{\partial^{\beta} u(x, t)}{\partial|x|^{\beta}}=-\frac{1}{2 \cos (\pi \beta / 2) \Gamma(2-\beta)} \frac{d^{2}}{d x^{2}} \int_{-\infty}^{\infty}|x-\xi|^{1-\beta} u(\xi, t) d \xi, \quad 1<\beta \leq 2,
$$

and $\Gamma(\cdot)$ denotes the gamma function.

There are several ways to approximate the Riesz fractional derivative. At first, the Riesz fractional derivative was generally approached by the Grünwald-Letnikov derivative approximation with the first order of accuracy [28]. In order to improve the convergence order, various mathematical methods, such as Richardson extrapolation method, the method of lines, fractional central difference method, the matrix transform method and other high order algorithms, were developed and applied to numerically solve the Riesz fractional diffusion equation, one can see $[27,29-33]$ and the references therein. Here we use the second order fractional central difference method provided in $[27,34]$ to discrete the Riesz fractional derivative of equation (1.1). It is interesting to note that in the case of $\lambda=0$, the time-space tempered fractional Fokker-Planck equation (1.1) reduces to the time and space fractional Fokker-Planck equation with Riesz fractional derivative. And the numerical solutions of the fractional Fokker-Planck equations have recently been presented in a number of works. Many works have been done on the study of numerical methods for time fractional Fokker-Planck equations [35-38] and space fractional Fokker-Planck equations [39-41]. There are some efficient computational techniques for numerically solving the space and time fractional Fokker-Planck equations [42-44]. However, very few theoretical results were provided to validate the effectiveness of the presented numerical methods. Deng [45] developed the finite element method for numerical solutions of the time and space-symmetric fractional Fokker-Planck equation, the theoretical analysis of stability and convergence are rigorously established. They proved that the obtained numerical scheme has $(2-\alpha)$ order accuracy in time direction and numerically verified by extensive experiments. Deng et al. [46] proposed the finite difference/predictor-corrector approach for the time and space-symmetric fractional Fokker- Planck equation, their theoretical analysis and numerical experiment results show that the numerical scheme is stable and converges with order $O\left(h+\tau^{\min \{1+2 \alpha, 2\}}\right)$.

So far, there have been only a limited number of works for the numerical solutions of the tempered fractional Fokker-Planck equations. Kullberg $[47,48]$ numerically solved the space tempered fractional Fokker-Planck equation with finite difference and spectral 
method. Gajda et al. [26, 49] established the numerical solution of the time tempered fractional Fokker-Planck equation via Monte Carlo methods. As far as we know, there are very few published papers for numerically solving the time-space tempered fractional Fokker-Planck equation. Therefore, the main objective of this paper is to design the high order numerical schemes for the time-space tempered fractional Fokker-Planck equation. The $q$ th $(q=1,2,3,4,5)$ order operators, named the tempered and weighted and shifted Lubich difference (TWSLD) operators, are used to approximate the tempered fractional derivative, and the classical central difference and fractional centered difference are used in the spatial discretization. The detailed theoretical analysis and numerical experiments are given to confirm the validity of the numerical schemes.

The outline of this paper is as follows. In Section 2, based on some definitions and properties of the tempered fractional calculus, we derive the equivalent form of Eq. (1.1). In Section 3, we apply TWSLD operators and central difference operators to approximate the time derivative and spatial derivatives, respectively. In Section 4, we perform the detailed theoretical analysis for the stability and convergence of the presented schemes. Extensive numerical experiments are carried out in Section 5 to confirm the theoretical results of our schemes. In Section 6, the probability density distribution of diffusion particles is simulated. We conclude the paper in the last section.

\section{The equivalent equation of Eq. (1.1)}

In this section, using the definitions and properties of fractional calculus, we transform Eq. (1.1) into another equivalent form, which is more convenient for approximating by our methods.

Using the definition of Riemann-Liouville tempered fractional derivative, Eq. (1.1) can be rewritten as

$$
\frac{\partial\left(e^{\lambda t} u(x, t)\right)}{\partial t}={ }_{0} D_{t}^{1-\alpha}\left[-\frac{\partial}{\partial x} F(x)+\frac{\partial^{\beta}}{\partial|x|^{\beta}}\right]\left(e^{\lambda t} u(x, t)\right) .
$$

Applying the operator ${ }_{0} D_{t}^{\alpha-1}$ (or ${ }_{0} I_{t}^{1-\alpha}$ ) on both sides of Eq. (2.1) leads to

$$
{ }_{0} D_{t}^{\alpha-1}\left[\frac{\partial\left(e^{\lambda t} u(x, t)\right)}{\partial t}\right]={ }_{0} D_{t}^{\alpha-1}\left\{{ }_{0} D_{t}^{1-\alpha}\left[-\frac{\partial}{\partial x} F(x)+\frac{\partial^{\beta}}{\partial|x|^{\beta}}\right]\left(e^{\lambda t} u(x, t)\right)\right\} .
$$

Recalling the composite properties of the Riemann-Liouville fractional calculus [5053], the left-hand side and the right-hand side of Eq. (2.2) can be rewritten as

$$
{ }_{0} D_{t}^{\alpha-1}\left[\frac{\partial\left(e^{\lambda t} u(x, t)\right)}{\partial t}\right]={ }_{0} D_{t}^{\alpha}\left(e^{\lambda t} u(x, t)\right)-\frac{t^{-\alpha}}{\Gamma(1-\alpha)} u(x, 0),
$$

and

$$
\begin{aligned}
{ }_{0} D_{t}^{\alpha-1} & \left\{{ }_{0} D_{t}^{1-\alpha}\left[-\frac{\partial}{\partial x} F(x)+\frac{\partial^{\beta}}{\partial|x|^{\beta}}\right]\left(e^{\lambda t} u(x, t)\right)\right\} \\
= & {\left[-\frac{\partial}{\partial x} F(x)+\frac{\partial^{\beta}}{\partial|x|^{\beta}}\right]\left(e^{\lambda t} u(x, t)\right) } \\
& -{ }_{0} D_{t}^{-\alpha}\left\{\left.\left[-\frac{\partial}{\partial x} F(x)+\frac{\partial^{\beta}}{\partial|x|^{\beta}}\right]\left(e^{\lambda t} u(x, t)\right)\right|_{t=0}\right\} \frac{t^{-\alpha}}{\Gamma(1-\alpha)} .
\end{aligned}
$$


If the function $u(t)$ is continuously differentiable in the interval $[0, t]$, then

$$
\begin{aligned}
\left.{ }_{0} D_{t}^{-\alpha}\left(e^{\lambda t} u(t)\right)\right|_{t=0} & =\lim _{t \rightarrow 0^{+}} \frac{1}{\Gamma(\alpha)} \int_{0}^{t}(t-\tau)^{\alpha-1} e^{\lambda \tau} u(\tau) d \tau \\
& =\lim _{t \rightarrow 0^{+}}\left[\frac{t^{\alpha} u(0)}{\Gamma(\alpha+1)}+\frac{1}{\Gamma(\alpha+1)} \int_{0}^{t}(t-\tau)^{\alpha} e^{\lambda \tau}\left(\lambda u(\tau)+\frac{d(u(\tau))}{d \tau}\right) d \tau\right] \\
& =0
\end{aligned}
$$

Since $u(x, t) \in C_{x, t}^{2,1}([a, b] \times[0, T])$, we can obtain

$$
{ }_{0} D_{t}^{-\alpha}\left\{\left.\left[-\frac{\partial}{\partial x} F(x)+\frac{\partial^{\beta}}{\partial|x|^{\beta}}\right]\left(e^{\lambda t} u(x, t)\right)\right|_{t=0}\right\}=0
$$

From above Eqs. (2.2)-(2.6), we get

$$
{ }_{0} D_{t}^{\alpha}\left(e^{\lambda t} u(x, t)\right)-\frac{t^{-\alpha}}{\Gamma(1-\alpha)} u(x, 0)=\left[-\frac{\partial}{\partial x} F(x)+\frac{\partial^{\beta}}{\partial|x|^{\beta}}\right]\left(e^{\lambda t} u(x, t)\right)
$$

multiplying both sides of Eq. (2.7) by $e^{-\lambda t}$ and using the definition defined in (1.2), we have

$$
{ }_{0} D_{t}^{\alpha, \lambda}(u(x, t))-\frac{t^{-\alpha} e^{-\lambda t}}{\Gamma(1-\alpha)} u(x, 0)=\left[-\frac{\partial}{\partial x} F(x)+\frac{\partial^{\beta}}{\partial|x|^{\beta}}\right] u(x, t)
$$

The relationship between Riemann-Liouville and the Caputo tempered fractional derivatives shows

$$
{ }_{0} D_{t}^{\alpha, \lambda} u(x, t)-\frac{t^{-\alpha} e^{-\lambda t}}{\Gamma(1-\alpha)} u(x, 0)={ }_{0}^{C} D_{t}^{\alpha, \lambda} u(x, t)={ }_{0} D_{t}^{\alpha, \lambda}\left[u(x, t)-e^{-\lambda t} u(x, 0)\right]
$$

then the equivalent form of Eq. (1.1) can be rewritten as follows:

$$
{ }_{0} D_{t}^{\alpha, \lambda}\left[u(x, t)-e^{-\lambda t} u(x, 0)\right]=\left[-\frac{\partial}{\partial x} F(x)+\frac{\partial^{\beta}}{\partial|x|^{\beta}}\right] u(x, t) .
$$

\section{Numerical schemes of Eq. (2.10)}

In this section, we consider Eq. (2.10) in a finite domain $[a, b]$ subject to the initial condition

$$
u(x, 0)=\psi(x), \quad a \leq x \leq b
$$

and the homogeneous Dirichlet boundary conditions

$$
u(a, t)=0, \quad u(b, t)=0, \quad 0<t \leq T .
$$

Note that the non-zero boundary conditions can be homogenized mathematically. 


\subsection{TWSLD operators and the fractional centered difference operator}

In this subsection, we derive the $q$ th order $(q \leq 5)$ operators, named TWSLD operators, by the corresponding coefficients of the generating functions $\Re^{q, \alpha}(\zeta)$ to approximate the Riemann-Liouville tempered fractional derivative of order $\alpha$. The form of the generating functions is as follows:

$$
\mathfrak{R}^{q, \alpha}(\zeta)=\left(\sum_{i=1}^{q} \frac{1}{i}\left(1-e^{-\lambda \tau} \zeta\right)^{i}\right)^{\alpha}, \quad q=1,2,3,4,5 .
$$

For $\lambda=0$, formula (3.3) reduces to the fractional Lubich methods. For $\lambda=0, \alpha=1$, the scheme reduces to the classical $(q+1)$-point backward difference formula [50]. We can also write formula (3.3) as the following form:

$$
\mathfrak{R}^{q, \alpha}(\zeta)=\left(\sum_{i=1}^{q} \frac{1}{i}\left(1-e^{-\lambda \tau} \zeta\right)^{i}\right)^{\alpha}=\sum_{k=0}^{\infty} d_{k}^{q, \alpha} \xi^{k}
$$

Then, for the Riemann-Liouville tempered fractional derivative, there is

$$
{ }_{0} D_{t}^{\alpha, \lambda} u\left(t_{n}\right)=\tau^{-\alpha} \sum_{k=0}^{n} d_{k}^{q, \alpha} u\left(t_{n-k}\right)+O\left(\tau^{q}\right), \quad q=1,2,3,4,5,
$$

where $\tau$ is the step size, $t_{n}=n \tau, d_{k}^{q, \alpha}$ can also be rewritten as

$$
d_{k}^{q, \alpha}=e^{-\lambda k \tau} l_{k}^{q, \alpha}, \quad q=1,2,3,4,5, k=0,1,2, \ldots, n .
$$

For more about the detailed description of $l_{k}^{q, \alpha}$, one can refer to [4].

Lemma 3.1 ([54]) For $q=1$, the coefficients $l_{k}^{q, \alpha}$ satisfy

$$
l_{0}^{1, \alpha}=1, \quad l_{k}^{1, \alpha}<0 \quad(k \geq 1), \quad \sum_{k=0}^{n-1} l_{k}^{1, \alpha}>0, \quad \sum_{k=0}^{\infty} l_{k}^{1, \alpha}=0,
$$

and

$$
\frac{1}{n^{\alpha} \Gamma(1-\alpha)}<\sum_{k=0}^{n-1} l_{k}^{1, \alpha}=-\sum_{k=n}^{\infty} l_{k}^{1, \alpha} \leq \frac{1}{n^{\alpha}} .
$$

Lemma 3.2 ([27]) Let $f \in C^{5}(R)$ and all derivatives up to order five belong to $L_{1}(R)$, and

$$
\Delta_{h}^{\beta} f(x)=\sum_{j=-\infty}^{\infty} \frac{(-1)^{j} \Gamma(\beta+1)}{\Gamma(\beta / 2-j+1) \Gamma(\beta / 2+j+1)} f(x-j h)
$$

be the fractional central difference. Then the Riesz fractional derivative for $1<\beta \leq 2$ can be approximated as

$$
\frac{\partial^{\beta} f(x)}{\partial|x|^{\beta}}=-\frac{\Delta_{h}^{\beta} f(x)}{h^{\beta}}+O\left(h^{2}\right) .
$$


If $\hat{f}$ is defined by

$$
\hat{f}(x)= \begin{cases}f(x), & x \in[a, b], \\ 0, & x \notin[a, b]\end{cases}
$$

such that $\hat{f}$ satisfies the conditions of Lemma 3.2, then we have

$$
\frac{\partial^{\beta} \hat{f}(x)}{\partial|x|^{\beta}}=-h^{-\beta} \sum_{j=-\infty}^{\infty} \frac{(-1)^{j} \Gamma(\beta+1)}{\Gamma(\beta / 2-j+1) \Gamma(\beta / 2+j+1)} \hat{f}(x-j h)+O\left(h^{2}\right) .
$$

Since $\hat{f}(x)=0$ for $x \notin[a, b]$, then

$$
\frac{\partial^{\beta} f(x)}{\partial|x|^{\beta}}=-h^{-\beta} \sum_{j=-\frac{b-x}{h}}^{\frac{x-a}{h}} \frac{(-1)^{j} \Gamma(\beta+1)}{\Gamma(\beta / 2-j+1) \Gamma(\beta / 2+j+1)} f(x-j h)+O\left(h^{2}\right),
$$

where $h=(b-a) / M$, and $M$ is the number of partitions of the interval $[a, b]$.

\subsection{Derivation of numerical schemes}

Let the mesh points $x_{i}=a+i h(i=0, \ldots, M)$ and $t_{n}=n \tau(n=0,1,2, \ldots, N)$, where $\tau=T / N$ and $h=(b-a) / M$ are the equidistant time step length and space step size, respectively.

Using the TWSLD operators (3.5) to approximate the Riemann-Liouville tempered fractional derivative ${ }_{0} D_{t}^{\alpha, \lambda} u(x, t)$ and ${ }_{0} D_{t}^{\alpha, \lambda}\left(e^{-\lambda t} u(x, 0)\right)$, we arrive at

$$
\begin{aligned}
& \left.{ }_{0} D_{t}^{\alpha, \lambda} u(x, t)\right|_{\left(x_{i}, t_{n}\right)}=\tau^{-\alpha} \sum_{k=0}^{n} d_{k}^{q, \alpha} u\left(x_{i}, t_{n-k}\right)+O\left(\tau^{q}\right), \quad q=1,2,3,4,5, \\
& { }_{0} D_{t}^{\alpha, \lambda}\left[e^{-\lambda t} u(x, 0)\right]_{\left(x_{i}, t_{n}\right)}=\tau^{-\alpha} \sum_{k=0}^{n} d_{k}^{q, \alpha} e^{-\lambda(n-k) \tau} u\left(x_{i}, 0\right)+O\left(\tau^{q}\right), \quad q=1,2,3,4,5 .
\end{aligned}
$$

To approximate Eq. (2.10), we apply the classical central difference formula and fractional centered difference formula (3.10) for the spatial derivatives, that is,

$$
\begin{aligned}
& \left.\frac{\partial}{\partial x}(-F(x) u(x, t))\right|_{\left(x_{i}, t_{n}\right)}=-\frac{F\left(x_{i+1}\right) u\left(x_{i+1}, t_{n}\right)-F\left(x_{i-1}\right) u\left(x_{i-1}, t_{n}\right)}{2 h}+O\left(h^{2}\right), \\
& \frac{\partial^{\beta} u\left(x_{i}, t_{n}\right)}{\partial|x|^{\beta}}=-h^{-\beta} \sum_{j=-M+i}^{i} g_{j} u\left(x_{i-j}, t_{n}\right)+O\left(h^{2}\right),
\end{aligned}
$$

where

$$
g_{j}=\frac{(-1)^{j} \Gamma(\beta+1)}{\Gamma(\beta / 2-j+1) \Gamma(\beta / 2+j+1)} .
$$


Combining Eqs. (3.11)-(3.14) and (3.6), we can obtain the following equation:

$$
\begin{aligned}
\tau^{-\alpha}\left[\sum_{k=0}^{n} e^{-\lambda k \tau} l_{k}^{q, \alpha} u\left(x_{i}, t_{n-k}\right)-e^{-\lambda n \tau} \sum_{k=0}^{n} l_{k}^{q, \alpha} u\left(x_{i}, 0\right)\right] \\
=-\frac{F\left(x_{i+1}\right) u\left(x_{i+1}, t_{n}\right)-F\left(x_{i-1}\right) u\left(x_{i-1}, t_{n}\right)}{2 h}-\frac{1}{h^{\beta}} \sum_{j=-M+i}^{i} g_{j} u\left(x_{i-j}, t_{n}\right)+r_{i}^{n},
\end{aligned}
$$

with

$$
\left|r_{i}^{n}\right| \leq C\left(\tau^{q}+h^{2}\right), \quad q=1,2,3,4,5
$$

where $C$ is a positive constant independent of $\tau$ and $h$.

Multiplying both sides of Eq. (3.16) by $\tau^{\alpha}$ results in

$$
\begin{aligned}
& l_{0}^{q, \alpha} u\left(x_{i}, t_{n}\right)+\sum_{k=1}^{n-1} e^{-\lambda k \tau} l_{k}^{q, \alpha} u\left(x_{i}, t_{n-k}\right)-e^{-\lambda n \tau} \sum_{k=0}^{n-1} l_{k}^{q, \alpha} u\left(x_{i}, 0\right) \\
& =-\tau^{\alpha} \frac{F\left(x_{i+1}\right) u\left(x_{i+1}, t_{n}\right)-F\left(x_{i-1}\right) u\left(x_{i-1}, t_{n}\right)}{2 h}-\frac{\tau^{\alpha}}{h^{\beta}} \sum_{j=-M+i}^{i} g_{j} u\left(x_{i-j}, t_{n}\right)+R_{i}^{n},
\end{aligned}
$$

with

$$
\left|R_{i}^{n}\right|=\left|\tau^{\alpha} r_{i}^{n}\right| \leq C \tau^{\alpha}\left(\tau^{q}+h^{2}\right), \quad q=1,2,3,4,5
$$

Denoting $u_{i}^{n}$ as the numerical approximation of $u\left(x_{i}, t_{n}\right)$ and omitting the local truncation errors $R_{i}^{n}$, we obtain the numerical schemes of Eq. (2.10) as follows:

$$
\begin{aligned}
& l_{0}^{q, \alpha} u_{i}^{n}+\sum_{k=1}^{n-1} e^{-\lambda k \tau} l_{k}^{q, \alpha} u_{i}^{n-k}-e^{-\lambda n \tau} \sum_{k=0}^{n-1} l_{k}^{q, \alpha} u_{i}^{0}=-\tau^{\alpha} \frac{F_{i+1} u_{i+1}^{n}-F_{i-1} u_{i-1}^{n}}{2 h}-\frac{\tau^{\alpha}}{h^{\beta}} \sum_{j=-M+i}^{i} g_{j} u_{i-j}^{n}, \\
& i=1,2, \ldots, M-1, n \geq 1
\end{aligned}
$$

or

$$
\begin{aligned}
& \frac{\tau^{\alpha}}{h^{\beta}} \sum_{j=-M+i}^{i} g_{j} u_{i-j}^{n}-\tau^{\alpha} \frac{F_{i-1}}{2 h} u_{i-1}^{n}+l_{0}^{q, \alpha} u_{i}^{n}+\tau^{\alpha} \frac{F_{i+1}}{2 h} u_{i+1}^{n}=e^{-\lambda n \tau} \sum_{k=0}^{n-1} l_{k}^{q, \alpha} u_{i}^{0}-\sum_{k=1}^{n-1} e^{-\lambda k \tau} l_{k}^{q, \alpha} u_{i}^{n-k}, \\
& \quad i=1,2, \ldots, M-1, n \geq 1 .
\end{aligned}
$$

The discrete schemes of (3.1)-(3.2) are given by (3.22)-(3.23), respectively

$$
\begin{aligned}
& u_{i}^{0}=\psi\left(x_{i}\right), \quad 0 \leq i \leq M, \\
& u_{0}^{n}=0, \quad u_{M}^{n}=0, \quad n \geq 1 .
\end{aligned}
$$




\section{Stability and convergence analysis}

In this section, we prove the stability and convergence of schemes (3.21) for $q=1$ by the discrete $L_{\infty}$ norm. We first introduce several lemmas that will be used later.

Lemma 4.1 ([27]) The coefficients $g_{j}$ defined in Eq. (3.15) for $j=0, \pm 1, \pm 2, \ldots, \beta>-1$, satisfy
(1) $g_{0} \geq 0$,
(2) $g_{-j}=g_{j} \leq 0 \quad$ for all $|j| \geq 1$,
(3) $\sum_{j=-\infty}^{\infty} g_{j}=0$ and $\sum_{\substack{j=-M+i \\ j \neq 0}}^{i} g_{j} \leq g_{0}$.

Lemma 4.2 ([55]) Let $\left\{u_{i}\right\}_{i=0}^{M}$ be the grid functions defined on grid $\bar{\Omega}=\left\{x_{i}\right\}_{i=0}^{M}$. For the difference operator equation

$$
\left\{\begin{array}{l}
\mathcal{L}_{h} u_{i}:=a_{i} u_{i}-\sum_{p \neq i} a_{p} u_{p}=\varphi_{i}, \quad x_{i} \in \Omega=\left\{x_{i}\right\}_{i=1}^{M-1}, \\
u_{0}=0, \quad u_{M}=0
\end{array}\right.
$$

where $a_{i}>0$, and $d_{i}=a_{i}-\sum_{p \neq i} a_{p}>0, i=1,2, \ldots, M-1$, there exists

$$
\max _{1 \leq i \leq M-1}\left|u_{i}\right| \leq \max _{1 \leq i \leq M-1} \frac{\left|\varphi_{i}\right|}{d_{i}}
$$

Theorem 4.1 Let $F(x)$ be an increasing function in the interval $[a, b]$ and $\lambda \geq 0$, then the numerical scheme (3.21) is unconditionally stable, i.e.,

$$
\left\|\varepsilon^{n}\right\|_{\infty} \leq\left\|\varepsilon^{0}\right\|_{\infty}, \quad n=1,2, \ldots, N
$$

Proof Let $v_{i}^{n}(i=0,1,2, \ldots, M, n=0,1,2, \ldots, N)$ be the approximate solution of scheme (3.21). Denote $\varepsilon^{n}=\left\{\varepsilon_{i}^{n}=v_{i}^{n}-u_{i}^{n} \mid 0 \leq i \leq M, n \geq 0\right\}$, then from Eq. (3.21) we get

$$
\begin{aligned}
& \frac{\tau^{\alpha}}{h^{\beta}} \sum_{j=-M+i}^{i} g_{j} \varepsilon_{i-j}^{n}-\tau^{\alpha} \frac{F_{i-1}}{2 h} \varepsilon_{i-1}^{n}+l_{0}^{1, \alpha} \varepsilon_{i}^{n}+\tau^{\alpha} \frac{F_{i+1}}{2 h} \varepsilon_{i+1}^{n}=e^{-\lambda n \tau} \sum_{k=0}^{n-1} l_{k}^{1, \alpha} \varepsilon_{i}^{0}-\sum_{k=1}^{n-1} e^{-\lambda k \tau} l_{k}^{1, \alpha} \varepsilon_{i}^{n-k} \\
& \quad i=0,1,2, \ldots, M, n \geq 1
\end{aligned}
$$

since $\varepsilon_{0}^{n}=0, \varepsilon_{M}^{n}=0$ and $l_{0}^{1, \alpha}=1$, we obtain

$$
\begin{aligned}
& \varepsilon_{i}^{n}+\frac{\tau^{\alpha} F_{i+1}}{2 h} \varepsilon_{i+1}^{n}-\frac{\tau^{\alpha} F_{i-1}}{2 h} \varepsilon_{i-1}^{n}+\frac{\tau^{\alpha}}{h^{\beta}} \sum_{j=-M+i+1}^{i-1} g_{j} \varepsilon_{i-j}^{n}=e^{-\lambda n \tau} \sum_{k=0}^{n-1} l_{k}^{1, \alpha} \varepsilon_{i}^{0}-\sum_{k=1}^{n-1} e^{-\lambda k \tau} l_{k}^{1, \alpha} \varepsilon_{i}^{n-k}, \\
& i=1,2, \ldots, M-1, n \geq 1 .
\end{aligned}
$$

According to Lemma 4.1, we have

$$
0=\sum_{j=-\infty}^{\infty} g_{j}=\sum_{j=-\infty}^{-M+i} g_{j}+\sum_{j=-M+i+1}^{i-1} g_{j}+\sum_{j=i}^{\infty} g_{j}, \quad i=1,2, \ldots, M-1,
$$


and $g_{-j}=g_{j} \leq 0$ for all $|j| \geq 1$, then $\sum_{j=-\infty}^{-M+i} g_{j} \leq 0, \sum_{j=i}^{\infty} g_{j} \leq 0$, we can further get

$$
\sum_{j=-M+i+1}^{i-1} g_{j} \geq 0, \quad i=1,2, \ldots, M-1 .
$$

Since $F(x)$ is an increasing function, we can easily obtain

$$
\left(1+\frac{\tau^{\alpha}}{h^{\beta}} g_{0}\right)+\frac{\tau^{\alpha}}{2 h}\left(F_{i+1}-F_{i-1}\right)+\frac{\tau^{\alpha}}{h^{\beta}} \sum_{\substack{j=-M+i+1 \\ j \neq 0}}^{i-1} g_{j} \geq 1, \quad i=1,2, \ldots, M-1
$$

From Lemma 4.2, it yields

$$
\begin{aligned}
\max _{1 \leq i \leq M-1}\left|\varepsilon_{i}^{n}\right| & \leq \max _{1 \leq i \leq M-1} \frac{\left|e^{-\lambda n \tau} \sum_{k=0}^{n-1} l_{k}^{1, \alpha} \varepsilon_{i}^{0}-\sum_{k=1}^{n-1} e^{-\lambda k \tau} l_{k}^{1, \alpha} \varepsilon_{i}^{n-k}\right|}{1+\frac{\tau^{\alpha}}{2 h}\left(F_{i+1}-F_{i-1}\right)+\frac{\tau^{\alpha}}{h^{\beta}} \sum_{j=-M+i+1}^{i-1} g_{j}} \\
& \leq \max _{1 \leq i \leq M-1}\left|e^{-\lambda n \tau} \sum_{k=0}^{n-1} l_{k}^{1, \alpha} \varepsilon_{i}^{0}-\sum_{k=1}^{n-1} e^{-\lambda k \tau} l_{k}^{1, \alpha} \varepsilon_{i}^{n-k}\right| .
\end{aligned}
$$

Combining $\sum_{k=0}^{n-1} l_{k}^{1, \alpha}>0, \sum_{k=1}^{n-1} l_{k}^{1, \alpha}<0, e^{-\lambda n \tau} \in(0,1]$ and inequality (4.10), we obtain

$$
\begin{aligned}
\max _{1 \leq i \leq M-1}\left|\varepsilon_{i}^{n}\right| & \leq \max _{1 \leq i \leq M-1}\left|e^{-\lambda n \tau} \sum_{k=0}^{n-1} l_{k}^{1, \alpha} \varepsilon_{i}^{0}\right|+\max _{1 \leq i \leq M-1}\left|\sum_{k=1}^{n-1} e^{-\lambda k \tau} l_{k}^{1, \alpha} \varepsilon_{i}^{n-k}\right| \\
& \leq \sum_{k=0}^{n-1} l_{k}^{1, \alpha}\left(\max _{1 \leq i \leq M-1}\left|\varepsilon_{i}^{0}\right|\right)-\sum_{k=1}^{n-1} l_{k}^{1, \alpha}\left(\max _{1 \leq i \leq M-1}\left|\varepsilon_{i}^{n-k}\right|\right)
\end{aligned}
$$

i.e.,

$$
\left\|\varepsilon^{n}\right\|_{\infty} \leq \sum_{k=0}^{n-1} l_{k}^{1, \alpha}\left\|\varepsilon^{0}\right\|_{\infty}-\sum_{k=1}^{n-1} l_{k}^{1, \alpha}\left\|\varepsilon^{n-k}\right\|_{\infty} .
$$

Next we prove the following estimate by mathematical induction:

$$
\left\|\varepsilon^{n}\right\|_{\infty} \leq\left\|\varepsilon^{0}\right\|_{\infty}
$$

For $n=1$, from inequality (4.12), we can see (4.13) holds obviously. Assuming

$$
\left\|\varepsilon^{k}\right\|_{\infty} \leq\left\|\varepsilon^{0}\right\|_{\infty}, \quad k=1,2, \ldots, n-1
$$

and using inequality (4.12), we obtain

$$
\begin{aligned}
\left\|\varepsilon^{n}\right\|_{\infty} & \leq \sum_{k=0}^{n-1} l_{k}^{1, \alpha}\left\|\varepsilon^{0}\right\|_{\infty}-\sum_{k=1}^{n-1} l_{k}^{1, \alpha}\left\|\varepsilon^{n-k}\right\|_{\infty} \\
& \leq \sum_{k=0}^{n-1} l_{k}^{1, \alpha}\left\|\varepsilon^{0}\right\|_{\infty}-\sum_{k=1}^{n-1} l_{k}^{1, \alpha}\left\|\varepsilon^{0}\right\|_{\infty}=\left\|\varepsilon^{0}\right\|_{\infty} .
\end{aligned}
$$

Hence, $\left\|\varepsilon^{n}\right\|_{\infty} \leq\left\|\varepsilon^{0}\right\|_{\infty}$, i.e., scheme (3.21) is unconditionally stable. 
Lemma 4.3 ([54]) Let $R \geq 0, a_{k} \geq 0, k=0,1, \ldots, N$, and satisfy

$$
a_{n} \leq-\sum_{k=1}^{n-1} l_{k}^{q, \alpha} a_{n-k}+R, \quad n \geq 1,
$$

(a) when $0<\alpha<1$,

$$
a_{n} \leq\left(\sum_{k=0}^{n-1} l_{k}^{q, \alpha}\right)^{-1} R \leq n^{\alpha} \Gamma(1-\alpha) R,
$$

(b) when $\alpha \rightarrow 1$,

$$
a_{n} \leq n R \text {. }
$$

Theorem 4.2 Let $F(x)$ be an increasing function in the interval $[a, b]$ and $\lambda \geq 0$, then the numerical scheme (3.21) is convergent, and the following error estimates hold:

$$
\left\|u\left(x_{i}, t_{n}\right)-u_{i}^{n}\right\|_{\infty} \leq C \Gamma(1-\alpha) T^{\alpha}\left(\tau+h^{2}\right), \quad \text { for } 0<\alpha<1,
$$

and

$$
\left\|u\left(x_{i}, t_{n}\right)-u_{i}^{n}\right\|_{\infty} \leq C T\left(\tau+h^{2}\right), \quad \text { for } \alpha \rightarrow 1,
$$

where the constant $C>0$ is independent of $\tau$ and $h$.

Proof Let $u\left(x_{i}, t_{n}\right)$ and $u_{i}^{n}$ be the exact solution and the numerical solution at $\left(x_{i}, t_{n}\right)$, respectively, and $e^{n}=\left\{e_{i}^{n}=u\left(x_{i}, t_{n}\right)-u_{i}^{n} \mid 0 \leq i \leq M, 0 \leq n \leq N\right\}$. Notice that

$$
\begin{aligned}
& e_{i}^{0}=0, \quad 1 \leq i \leq M-1, \\
& e_{0}^{n}=0, \quad e_{M}^{n}=0, \quad 0 \leq n \leq N .
\end{aligned}
$$

Subtracting Eq. (3.18) from Eq. (3.21), we can get the following error equation:

$$
\begin{aligned}
& e_{i}^{n}+\frac{\tau^{\alpha} F_{i+1}}{2 h} e_{i+1}^{n}-\frac{\tau^{\alpha} F_{i-1}}{2 h} e_{i-1}^{n}+\frac{\tau^{\alpha}}{h^{\beta}} \sum_{j=-M+i+1}^{i-1} g_{j} e_{i-j}^{n}=-\sum_{k=1}^{n-1} e^{-\lambda k \tau} l_{k}^{1, \alpha} e_{i}^{n-k}+R_{i}^{n}, \\
& \quad i=1,2, \ldots, M-1, n \geq 1,
\end{aligned}
$$

where $R_{i}^{n}$ is defined by (3.19). From Lemma 4.2, we derive

$$
\begin{aligned}
\max _{1 \leq i \leq M-1}\left|e_{i}^{n}\right| & \leq \max _{1 \leq i \leq M-1} \frac{\left|-\sum_{k=1}^{n-1} e^{-\lambda k \tau} l_{k}^{1, \alpha} e_{i}^{n-k}+R_{i}^{n}\right|}{1+\frac{\tau^{\alpha}}{2 h}\left(F_{i+1}-F_{i-1}\right)+\frac{\tau^{\alpha}}{h^{\beta}} \sum_{j=-M+i}^{i} g_{j}} \\
& \leq \max _{1 \leq i \leq M-1}\left|-\sum_{k=1}^{n-1} e^{-\lambda k \tau} l_{k}^{1, \alpha} e_{i}^{n-k}+R_{i}^{n}\right| \\
& \leq-\sum_{k=1}^{n-1} l_{k}^{1, \alpha}\left(\max _{1 \leq i \leq M-1}\left|e_{i}^{n-k}\right|\right)+R_{\max } .
\end{aligned}
$$


Using the definition of discrete $L_{\infty}$ norm leads to

$$
\left\|e^{n}\right\|_{\infty} \leq-\sum_{k=1}^{n-1} l_{k}^{1, \alpha}\left\|e^{n-k}\right\|_{\infty}+R_{\max }
$$

where $R_{\max }=\max _{1 \leq i \leq M-1, n \geq 1}\left|R_{i}^{n}\right|$.

Hence, according to Lemma 4.3, we have

$$
\begin{aligned}
& \left\|u\left(x_{i}, t_{n}\right)-u_{i}^{n}\right\|_{\infty} \leq C \Gamma(1-\alpha) T^{\alpha}\left(\tau+h^{2}\right), \quad 0<\alpha<1, \\
& \left\|u\left(x_{i}, t_{n}\right)-u_{i}^{n}\right\|_{\infty} \leq C T\left(\tau+h^{2}\right), \quad \alpha \rightarrow 1 .
\end{aligned}
$$

\section{Numerical examples}

In this section, two examples are used to illustrate the effectiveness of the algorithms and to verify the above theoretical results. The first example is used to testify the efficiency of the TWSLD operators, the second example is calculated by the presented numerical schemes (3.21), and the validity of our developed algorithms is confirmed.

Example 5.1 In this example, we consider the following tempered fractional ordinary differential initial value problem:

$$
\left\{\begin{array}{l}
{ }_{0} D_{t}^{\alpha, \lambda}\left[u(t)-e^{-\lambda t} u(0)\right]=e^{-\lambda t} \frac{\Gamma(4+\alpha)}{\Gamma(4)} t^{3}, \quad 0<\alpha<1, \\
u(0)=0
\end{array}\right.
$$

then the exact solution of this problem is $u(t)=e^{-\lambda t} t^{3+\alpha}$. The numerical results are shown in Tables 1-4. The errors are measured by the $L_{\infty}$ norm

$$
\operatorname{Err}(\tau)=\left\|u\left(t_{n}\right)-u^{n}\right\|_{\infty}=\max _{0 \leq n \leq N}\left|u\left(t_{n}\right)-u^{n}\right|,
$$

\begin{tabular}{|c|c|c|c|c|c|c|}
\hline \multirow[t]{2}{*}{$\tau$} & \multicolumn{2}{|l|}{$\alpha=0.1$} & \multicolumn{2}{|l|}{$\alpha=0.5$} & \multicolumn{2}{|l|}{$\alpha=0.9$} \\
\hline & $\operatorname{Err}(\tau)$ & Rate & $\operatorname{Err}(\tau)$ & Rate & $\operatorname{Err}(\tau)$ & Rate \\
\hline $1 / 20$ & $1.042402 \mathrm{e}-03$ & * & $5.951186 \mathrm{e}-03$ & * & $1.211901 \mathrm{e}-02$ & * \\
\hline $1 / 40$ & $5.228154 \mathrm{e}-04$ & 0.9955 & 2.968097e-03 & 1.0036 & 5.998746e-03 & 1.0145 \\
\hline $1 / 80$ & $2.618102 \mathrm{e}-04$ & 0.9978 & $1.482148 \mathrm{e}-03$ & 1.0018 & $2.984155 \mathrm{e}-03$ & 1.0073 \\
\hline $1 / 160$ & $1.310056 \mathrm{e}-04$ & 0.9989 & $7.405955 \mathrm{e}-04$ & 1.0009 & $1.488269 \mathrm{e}-03$ & 1.0037 \\
\hline $1 / 320$ & $6.552793 e-05$ & 0.9994 & $3.701777 \mathrm{e}-04$ & 1.0005 & $7.431822 \mathrm{e}-04$ & 1.0018 \\
\hline
\end{tabular}

and the convergent order is approximated by

$$
\text { Rate }=\log _{2} \frac{\operatorname{Err}(\tau)}{\operatorname{Err}(\tau / 2)}
$$

Table 1 Numerical errors and convergent orders with $\lambda=2, T=1$, when $q=1$ 
Table 2 Numerical errors and convergent orders with $\lambda=2, T=1$, when $q=2$

\begin{tabular}{|c|c|c|c|c|c|c|}
\hline \multirow[t]{2}{*}{$\tau$} & \multicolumn{2}{|l|}{$\alpha=0.1$} & \multicolumn{2}{|l|}{$\alpha=0.5$} & \multicolumn{2}{|l|}{$\alpha=0.9$} \\
\hline & $\overline{\operatorname{Err}}(\tau)$ & Rate & $\overline{\operatorname{Err}}(\tau)$ & Rate & $\operatorname{Err}(\tau)$ & Rate \\
\hline $1 / 20$ & 8.679529e-05 & * & $4.906284 \mathrm{e}-04$ & * & $1.069498 \mathrm{e}-03$ & * \\
\hline $1 / 40$ & $2.252822 \mathrm{e}-05$ & 1.9459 & $1.272612 \mathrm{e}-04$ & 1.9468 & $2.772513 e-04$ & 1.9477 \\
\hline $1 / 80$ & $5.738671 \mathrm{e}-06$ & 1.9729 & $3.241215 \mathrm{e}-05$ & 1.9732 & $7.060275 \mathrm{e}-05$ & 1.9734 \\
\hline $1 / 160$ & $1.448182 \mathrm{e}-06$ & 1.9865 & $8.179024 \mathrm{e}-06$ & 1.9865 & $1.781554 \mathrm{e}-05$ & 1.9866 \\
\hline $1 / 320$ & $3.637467 \mathrm{e}-07$ & 1.9932 & $2.054342 \mathrm{e}-06$ & 1.9933 & $4.474725 \mathrm{e}-06$ & 1.9933 \\
\hline
\end{tabular}

Table 3 Numerical errors and convergent orders with $\lambda=2, T=1$, when $q=3$

\begin{tabular}{|c|c|c|c|c|c|c|}
\hline \multirow[t]{2}{*}{$\tau$} & \multicolumn{2}{|l|}{$\alpha=0.1$} & \multicolumn{2}{|l|}{$\alpha=0.5$} & \multicolumn{2}{|l|}{$\alpha=0.9$} \\
\hline & $\operatorname{Err}(\tau)$ & Rate & $\operatorname{Err}(\tau)$ & Rate & $\operatorname{Err}(\tau)$ & Rate \\
\hline $1 / 20$ & $1.285963 \mathrm{e}-05$ & * & $5.527985 \mathrm{e}-05$ & $*$ & $1.062574 \mathrm{e}-04$ & $*$ \\
\hline $1 / 40$ & $1.777100 \mathrm{e}-06$ & 2.8553 & 7.323031e-06 & 2.9162 & $1.410216 \mathrm{e}-05$ & 2.9136 \\
\hline $1 / 80$ & $2.295725 \mathrm{e}-07$ & 2.9525 & $9.432216 \mathrm{e}-07$ & 2.9568 & $1.816285 \mathrm{e}-06$ & 2.9569 \\
\hline $1 / 160$ & $2.888395 \mathrm{e}-08$ & 2.9906 & $1.196761 \mathrm{e}-07$ & 2.9785 & $2.304527 \mathrm{e}-07$ & 2.9784 \\
\hline $1 / 320$ & $3.637465 \mathrm{e}-09$ & 2.9893 & $1.507136 \mathrm{e}-08$ & 2.9893 & $2.902244 \mathrm{e}-08$ & 2.9892 \\
\hline
\end{tabular}

Table 4 Numerical errors and convergent orders with $\lambda=2, T=1$, when $q=4$

\begin{tabular}{|c|c|c|c|c|c|c|}
\hline \multirow[t]{2}{*}{$\tau$} & \multicolumn{2}{|l|}{$\alpha=0.1$} & \multicolumn{2}{|l|}{$\alpha=0.5$} & \multicolumn{2}{|l|}{$\alpha=0.9$} \\
\hline & $\overline{\operatorname{Err}}(\tau)$ & Rate & $\operatorname{Err}(\tau)$ & Rate & $\operatorname{Err}(\tau)$ & Rate \\
\hline $1 / 20$ & $1.654609 \mathrm{e}-06$ & * & $6.172450 \mathrm{e}-06$ & * & $1.076598 \mathrm{e}-05$ & * \\
\hline $1 / 40$ & $1.490310 \mathrm{e}-07$ & 3.4728 & $4.637825 \mathrm{e}-07$ & 3.7343 & $7.508511 \mathrm{e}-07$ & 3.8418 \\
\hline $1 / 80$ & $1.286134 \mathrm{e}-08$ & 3.5345 & $3.288428 \mathrm{e}-08$ & 3.8180 & $4.965133 \mathrm{e}-08$ & 3.9186 \\
\hline $1 / 160$ & $1.085597 \mathrm{e}-09$ & 3.5665 & $2.255987 \mathrm{e}-09$ & 3.8656 & 3.194957e-09 & 3.9580 \\
\hline $1 / 320$ & $9.059726 \mathrm{e}-11$ & 3.5829 & $1.517684 \mathrm{e}-10$ & 3.8938 & $2.027282 \mathrm{e}-10$ & 3.9782 \\
\hline
\end{tabular}

Example 5.2 Without loss of generality, we add a force term $f(x, t)$ on the right-hand side of Eq. (2.10). We consider

$$
{ }_{0} D_{t}^{\alpha, \lambda}\left[u(x, t)-e^{-\lambda t} u(x, 0)\right]=\left[-\frac{\partial}{\partial x} F(x)+\frac{\partial^{\beta}}{\partial|x|^{\beta}}\right] u(x, t)+f(x, t),
$$

where $0<\alpha<1,1<\beta \leq 2, \lambda \geq 0,0<x<1,0<t \leq T$, with $F(x)=x^{2}$ and the homogeneous initial boundary conditions

$$
\begin{aligned}
& u(x, 0)=0, \quad 0 \leq x \leq 1, \\
& u(0, t)=0, \quad u(1, t)=0, \quad 0<t \leq T,
\end{aligned}
$$

and the force term $f(x, t)$ is

$$
\begin{aligned}
f(x, t)= & e^{-\lambda t} \frac{\Gamma(4)}{\Gamma(4-\alpha)} t^{3-\alpha} x^{2}(1-x)^{2}+e^{-\lambda t} t^{3} 2 x^{3}(1-x)(2-3 x) \\
& +\frac{e^{-\lambda t} t^{3}}{2 \cos \left(\frac{\pi \beta}{2}\right)}\left[\frac{\Gamma(5)}{\Gamma(5-\beta)}\left(x^{4-\beta}+(1-x)^{4-\beta}\right)\right. \\
& \left.-2 \frac{\Gamma(4)}{\Gamma(4-\beta)}\left(x^{3-\beta}+(1-x)^{3-\beta}\right)+\frac{\Gamma(3)}{\Gamma(3-\beta)}\left(x^{2-\beta}+(1-x)^{2-\beta}\right)\right] .
\end{aligned}
$$


The exact solution of this problem is

$$
u(x, t)=e^{-\lambda t} t^{3} x^{2}(1-x)^{2}
$$

Here, the error in the $L_{\infty}$ norm is denoted by

$$
\operatorname{Err}(\tau, h)=\left\|u\left(x_{i}, t_{n}\right)-u_{i}^{n}\right\|_{\infty}=\max _{0 \leq i \leq M}\left|u\left(x_{i}, t_{n}\right)-u_{i}^{n}\right| u\left(x_{i}, t_{n}\right)-u_{i}^{n} \mid, \quad n \geq 0 .
$$

For theoretical convergent accuracy $O\left(\tau^{q}+h^{p}\right)$, the convergent order about step length $h$ can be approximated by

$$
\text { Rate }=\log _{2} \frac{\operatorname{Err}(\tau, h)}{\operatorname{Err}\left(2^{-p / q} \tau, h / 2\right)} .
$$

Problem (5.2)-(5.3) is calculated by the new numerical schemes (3.21), and the numerical

\begin{tabular}{|c|c|c|c|c|c|c|}
\hline \multirow[t]{2}{*}{$h$} & \multicolumn{2}{|l|}{$\alpha=0.1$} & \multicolumn{2}{|l|}{$\alpha=0.5$} & \multicolumn{2}{|l|}{$\alpha=0.9$} \\
\hline & $\operatorname{Err}(h, \tau)$ & Rate & $\overline{\operatorname{Err}(h, \tau)}$ & Rate & $\operatorname{Err}(h, \tau)$ & Rate \\
\hline $1 / 10$ & $1.671032 \mathrm{e}-04$ & * & $1.640323 e-04$ & * & $1.629271 \mathrm{e}-04$ & * \\
\hline $1 / 20$ & $3.928033 e-05$ & 2.0889 & $3.880801 \mathrm{e}-05$ & 2.0796 & $3.890401 \mathrm{e}-05$ & 2.0662 \\
\hline $1 / 40$ & $9.352858 \mathrm{e}-06$ & 2.0703 & $9.296651 \mathrm{e}-06$ & 2.0616 & $9.399219 \mathrm{e}-06$ & 2.0493 \\
\hline $1 / 80$ & $2.245405 \mathrm{e}-06$ & 2.0584 & $2.244103 e-06$ & 2.0506 & $2.286094 \mathrm{e}-06$ & 2.0397 \\
\hline
\end{tabular}
results with different parameters $\alpha, \beta$ and $\lambda$ are given in Tables 5-8. It is obvious that

Table 5 Numerical errors and convergent orders with $\beta=1.5, \lambda=2, T=1$, when $q=1, \tau=h^{2}$

\begin{tabular}{|c|c|c|c|c|c|c|}
\hline \multirow[t]{2}{*}{$\boldsymbol{h}$} & \multicolumn{2}{|l|}{$\alpha=0.1$} & \multicolumn{2}{|l|}{$\alpha=0.5$} & \multicolumn{2}{|l|}{$\alpha=0.9$} \\
\hline & $\overline{\operatorname{Err}}(h, \tau)$ & Rate & $\overline{\operatorname{Err}}(h, \tau)$ & Rate & $\overline{\operatorname{Err}}(h, \tau)$ & Rate \\
\hline $1 / 10$ & $1.972781 \mathrm{e}-04$ & * & $1.806785 \mathrm{e}-04$ & $*$ & $1.523243 e-04$ & $*$ \\
\hline $1 / 20$ & $4.280811 \mathrm{e}-05$ & 2.2043 & $3.874839 \mathrm{e}-05$ & 2.2212 & $3.254807 e-05$ & 2.2265 \\
\hline $1 / 40$ & $1.011908 \mathrm{e}-05$ & 2.0808 & $9.293740 \mathrm{e}-06$ & 2.0598 & $8.656409 \mathrm{e}-06$ & 1.9107 \\
\hline $1 / 80$ & $2.226747 e-06$ & 2.1841 & $2.004849 e-06$ & 2.2128 & $1.715268 \mathrm{e}-06$ & 2.3353 \\
\hline
\end{tabular}

Table 6 Numerical errors and convergent orders with $\beta=1.5, \lambda=2, T=1$, when $q=4$, $\tau=h^{1 / 2}$

\begin{tabular}{|c|c|c|c|c|c|c|}
\hline \multirow[t]{2}{*}{$\boldsymbol{h}$} & \multicolumn{2}{|l|}{$\beta=1.1$} & \multicolumn{2}{|l|}{$\beta=1.5$} & \multicolumn{2}{|l|}{$\beta=1.9$} \\
\hline & $\operatorname{Err}(h, \tau)$ & Rate & $\operatorname{Err}(h, \tau)$ & Rate & $\operatorname{Err}(h, \tau)$ & Rate \\
\hline $1 / 10$ & $1.624398 \mathrm{e}-04$ & $*$ & $1.640323 e-04$ & $*$ & $2.552445 \mathrm{e}-04$ & $*$ \\
\hline $1 / 20$ & $5.330786 \mathrm{e}-05$ & 1.6075 & $3.880801 \mathrm{e}-05$ & 2.0796 & $6.242922 \mathrm{e}-05$ & 2.0316 \\
\hline $1 / 40$ & $1.371705 e-05$ & 1.9584 & $9.296651 \mathrm{e}-06$ & 2.0616 & $1.532306 \mathrm{e}-05$ & 2.0265 \\
\hline $1 / 80$ & $3.312170 \mathrm{e}-06$ & 2.0501 & $2.244103 e-06$ & 2.0506 & $3.765450 \mathrm{e}-06$ & 2.0248 \\
\hline
\end{tabular}

Table 7 Numerical errors and convergent orders with $\alpha=0.5, \lambda=2, T=1$, when $q=1, \tau=h^{2}$

\begin{tabular}{|c|c|c|c|c|c|c|}
\hline \multirow[t]{2}{*}{$\boldsymbol{h}$} & \multicolumn{2}{|l|}{$\lambda=1$} & \multicolumn{2}{|l|}{$\lambda=3$} & \multicolumn{2}{|l|}{$\lambda=5$} \\
\hline & $\operatorname{Err}(h, \tau)$ & Rate & $\operatorname{Err}(h, \tau)$ & Rate & $\operatorname{Err}(h, \tau)$ & Rate \\
\hline $1 / 20$ & $1.185249 \mathrm{e}-04$ & $*$ & $1.266770 \mathrm{e}-05$ & * & $1.353897 \mathrm{e}-06$ & * \\
\hline $1 / 40$ & $2.811036 \mathrm{e}-05$ & 2.0760 & $3.072661 \mathrm{e}-06$ & 2.0436 & $3.358635 \mathrm{e}-07$ & 2.0112 \\
\hline $1 / 80$ & $5.483807 e-06$ & 2.3579 & $7.329619 \mathrm{e}-07$ & 2.0677 & $9.796718 \mathrm{e}-08$ & 1.7775 \\
\hline $1 / 160$ & $1.396193 \mathrm{e}-06$ & 1.9737 & $1.787564 \mathrm{e}-07$ & 2.0357 & $2.288642 \mathrm{e}-08$ & 2.0978 \\
\hline
\end{tabular}

Table 8 Numerical errors and convergent orders with $\alpha=0.5, \beta=1.5, T=1$, when $q=4$, $\tau=h^{1 / 2}$ 
the numerical schemes with high order accuracy and the convergence orders are almost consistent with the theoretical results $O\left(\tau^{q}+h^{2}\right)$.

\section{Numerical simulations}

Using the numerical schemes (3.21), we simulate the probability density distribution $u(x, t)$ of the diffusion particles on a finite domain $[0,4]$. We consider the Gaussian function which tends to the $\delta$ function when $\sigma \rightarrow 0$ [7]

$$
u(x, 0)=\frac{1}{\sigma \sqrt{2 \pi}} \exp \left(-\frac{(x-2)^{2}}{2 \sigma^{2}}\right)
$$

as the initial condition, and $u(0, t)=u(4, t)=0$ as the boundary conditions. In the calculation process, let the force term $f(x, t)=0, h=1 / 40, \tau=h^{2}, \sigma=0.01$.

Let $F(x)=x$, the numerical results under different parameters are shown in Figure 1. It can be seen that the values of $\alpha, \beta, \lambda, t$ have a different effect on the probability density of the particles, but all of these curves have a cusp. The experimental results are in agreement with the analytic results given in $[56,57]$, which indicates the effectiveness of our numerical schemes again.

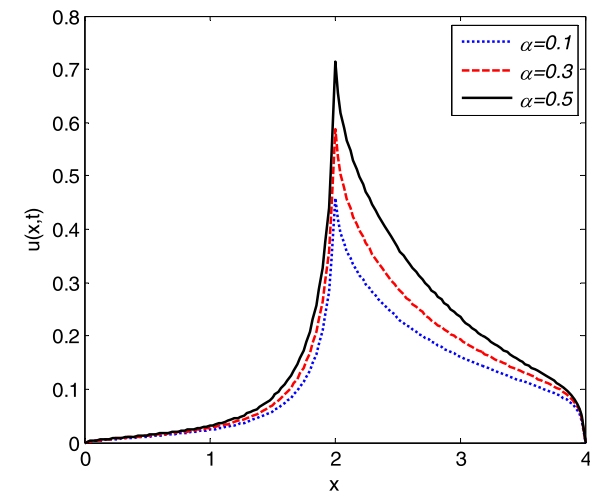

(a) $\beta=1.5, \lambda=2, t=0.1$

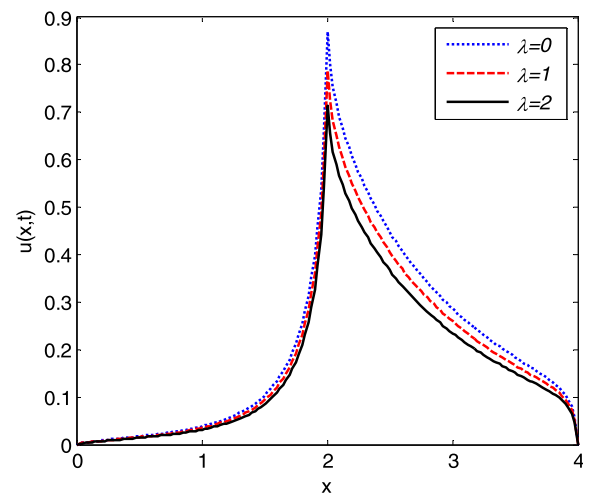

(c) $\alpha=0.5, \beta=1.5, t=0.1$

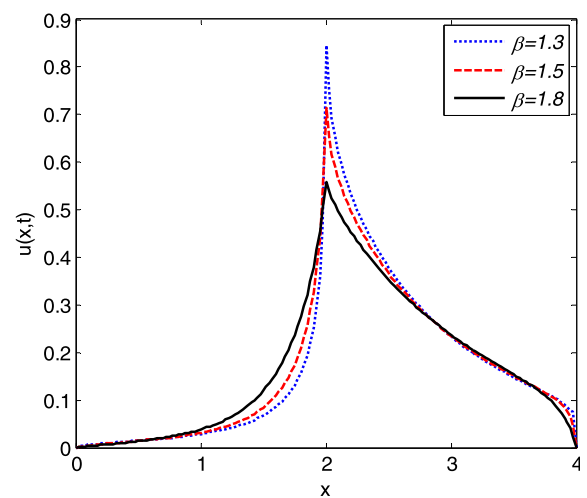

(b) $\alpha=0.5, \lambda=2, t=0.1$

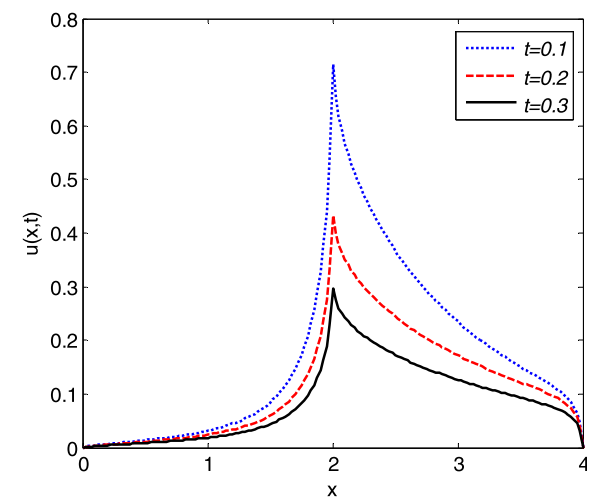

(d) $\alpha=0.5, \beta=1.5, \lambda=2$

Figure 1 The probability density distribution of particles under different values of $\alpha, \beta, \lambda, t$. 


\title{
7 Conclusions
}

It is well known that the time-space fractional Fokker-Planck equations play a key role in modelling relevant physical processes. The high order numerical algorithms for the differential equations naturally become an urgent topic. In this work, the high order schemes have been used to numerically solve the time-space tempered fractional Fokker-Planck equation with the space Riesz fractional derivative. We have proved theoretically that the numerical scheme is unconditionally stable and convergent with orders $O\left(\tau^{q}+h^{2}\right)$ $(q=1,2,3,4,5)$, which is higher than some recently studied schemes in terms of temporal direction. Extensive numerical experiments and simulations are carried out to verify the theoretical results.

\begin{abstract}
Acknowledgements
We would like to thank the anonymous referees for carefully reading this manuscript and for their valuable comments and constructive suggestions for improving this manuscript significantly. We are also very grateful to Dr. Can Li for his very helpful suggestions and providing us some references. This work was supported by the National Natural Science Foundation of China (No. 11472211) and Shaanxi science and technology research projects (No. 2015GY004). The authors gratefully acknowledge the support.
\end{abstract}

Abbreviations
TWSLD: tempered and weighted and shifted Lubich difference

Ethics approval and consent to participate

Not applicable

Competing interests

We declare that we have no competing interests.

Consent for publication

Not applicable

Authors' contributions

The authors have equal contributions to each part of this paper. All the authors read and approved the manuscript.

\section{Publisher's Note}

Springer Nature remains neutral with regard to jurisdictional claims in published maps and institutional affiliations.

Received: 17 May 2017 Accepted: 9 August 2017 Published online: 29 August 2017

\section{References}

1. Liu, FW, Zhuang, PH, Liu, QX: The Applications and Numerical Methods of Differential Equations. Science Press, Beijing (2015)

2. Sun, ZZ, Gao, GH: Numerical Methods for Fractional Differential Equations. Science Press, Beijing (2015)

3. Li, C, Zhao, S: Efficient numerical schemes for fractional water wave models. Comput. Math. Appl. 71, 238-254 (2016)

4. Chen, MH, Deng, WH: Fourth order difference approximations for space Riemann-Liouville derivatives based on weighted and shifted Lubich difference operators. Commun. Comput. Phys. 16, 516-540 (2014)

5. Li, C, Deng, WH: A new family of difference schemes for space fractional advection diffusion equation. Adv. Appl. Math. Mech. 9, 282-306 (2017)

6. Chen, $\mathrm{MH}$, Deng, WH: Fourth order accurate scheme for the space fractional diffusion equations. SIAM J. Numer. Anal. 52, 1418-1438 (2014)

7. Sousa, E, Li, C: A weighted finite difference method for the fractional diffusion equation based on the Riemann-Liouville derivative. Appl. Numer. Math. 90, 22-37 (2011)

8. Li, W, Li, C: Second-order explicit difference schemes for the space fractional advection diffusion equation. Appl. Math. Comput. 257, 446-457 (2015)

9. Baleanu, D, Mousalou, A, Rezapour, S: A new method for investigating approximate solutions of some fractional integro-differential equations involving the Caputo-Fabrizio derivative. Adv. Differ. Equ. 2017, 51 (2017)

10. Sakar, MG, Akgül, A, Baleanu, D: On solutions of fractional Riccati differential equations. Adv. Differ. Equ. 2017, 39 (2017)

11. Meerschaert, MM, Zhang, Y, Baeumer, B: Tempered anomalous diffusion in heterogeneous systems. Geophys. Res. Lett. 35, 254-268 (2008)

12. Meerschaert, MM, Sabzikar, F: Tempered fractional Brownian motion. Stat. Probab. Lett. 83, 2269-2275 (2014)

13. Baeumer, B, Meerschaert, MM: Tempered stable Lévy motion and transient super-diffusion. J. Comput. Appl. Math. 223, 2438-2448 (2010)

14. Sabzikar, F, Meerschaert, MM, Chen, J: Tempered fractional calculus. J. Comput. Phys. 293, 14-28 (2015)

15. $\mathrm{Li}, \mathrm{C}:$ On the stability of exact $A B C$ s for the reaction-subdiffusion equation on unbounded domain (30 Oct 2015) arXiv:1510.08761v2 [math.AP] 
16. Li, C, Deng, WH, Zhao, LJ: Well-posedness and numerical algorithm for the tempered fractional ordinary differential equations (2 Jan 2015) arXiv:1501.00376v1 [math.CA]

17. Li, C, Deng, WH: High order schemes for the tempered fractional diffusion equations. Adv. Comput. Math. 42, 543-572 (2016)

18. Metzler, R, Klafter, J: The random walk's guide to anomalous diffusion: a fractional dynamics approach. Phys. Rep. 339, 1-77 (2000)

19. $\mathrm{He}, J \mathrm{Q}, \mathrm{Dong}, \mathrm{Y}, \mathrm{Li}, \mathrm{ST}$, et al.: Study on force distribution of the tempered glass based on laser interference technology. Optik 126, 5276-5279 (2015)

20. Rosenau, P: Tempered diffusion: a transport process with propagating fronts and inertial delay. Phys. Rev. A 46 7371-7374 (1993)

21. Cartea, Á, del-Castillo-Negrete, D: Fractional diffusion models of option prices in markets with jumps. Physica A 374 749-763 (2006)

22. Hanyga, A: Wave propagation in media with singular memory. Math. Comput. Model. 34, 1399-1421 (2001)

23. Metzler, R, Barkai, E, Klafter, J: Anomalous diffusion and relaxation close to thermal equilibrium: a fractional Fokker-Planck equation approach. Phys. Rev. Lett. 82, 3563-3567 (1999)

24. Viswanathan, GM, Afanasyev, V, Buldyrev, SV, et al.: Lévy flight search patterns of wandering albatrosses. Nature 381, 413-415 (1996)

25. Cartea, Á, del-Castillo-Negrete, D: Fluid limit of the continuous-time random walk with general Lévy flight distribution functions. Phys. Rev. E 76 (2007)

26. Gajda, J, Magdziarz, M: Fractional Fokker-Planck equation with tempered $\alpha$-stable waiting times: Langevin picture and computer simulation. Phys. Rev. E 82, 011117 (2010)

27. Çelik, C, Duman, M: Crank-Nicolson method for the fractional diffusion equation with the Riesz fractional derivative. J. Comput. Phys. 231, 1743-1750 (2012)

28. Shen, S, Liu, F, Anh, V: Numerical approximations and solution techniques for the space-time Riesz-Caputo fractiona advection-diffusion equation. Numer. Algorithms 56, 383-403 (2011)

29. Ding, HF, Li, CP, Chen, YQ: High-order algorithms for Riesz derivative and their applications (II). J. Comput. Phys. 293, 218-237 (2015)

30. Ding, HF, Li, CP, Chen, YQ: High-order algorithms for Riesz derivative and their applications (III). Fract. Calc. Appl. Anal. 19, 19-55 (2016)

31. Yang, QQ, Turner, I, Liu, F: Analytical and numerical solutions for the time and space-symmetric fractional diffusion equation. ANZIAM J. Electron. Suppl. 50, C800-C814 (2009)

32. Wu, GC, Baleanu, D, Deng, ZG, Zeng, SD: Lattice fractional diffusion equation in terms of a Riesz-Caputo difference. Physica A 438, 335-339 (2015)

33. Wu, GC, Baleanu, D, Xie, HP: Riesz Riemann-Liouville difference on discrete domains. Chaos 26, 084308 (2016)

34. Ortigueira, MD: Riesz potential operators and inverses via fractional centred derivatives. Int. J. Math. Math. Sci. 2006, Article ID 48391 (2006)

35. Deng, WH: Numerical algorithm for the time fractional Fokker-Planck equation. J. Comput. Phys. 227, 1510-1522 (2007)

36. Vong, S, Wang, Z: A high order compact finite difference scheme for time fractional Fokker-Planck equations. Appl. Math. Lett. 43, 38-43 (2015)

37. Chen, S, Liu, F, et al.: Finite difference approximations for the fractional Fokker-Planck equation. Appl. Math. Model. 33, 256-273 (2009)

38. Le, KN, Mclean, W, Mustapha, K: Numerical solution of the time-fractional Fokker-Planck equation with general forcing. SIAM J. Numer Anal. 54, 1763-1784 (2016)

39. Zhao, Z, Li, CP: The finite element method for the generalized space fractional Fokker-Planck equation. In: IEEE/ASME MESA, pp. 511-516 (2010)

40. Zhang, Y: Padé approximation method for solving space fractional Fokker-Planck equations. Appl. Math. Lett. 35, 109-114 (2014)

41. Hafez, RM, Ezz-Eldien, SS, Bhrawy, AH, Ahmed, EA, Baleanu, D: A Jacobi Gauss-Lobatto and Gauss-Radau collocation algorithm for solving fractional Fokker-Planck equations. Nonlinear Dyn. 82, 1431-1440 (2015)

42. Yan, L: Numerical solutions of fractional Fokker-Planck equations using iterative Laplace transform method. Abstr. Appl. Anal. 2013, 465160 (2013)

43. Baleanu, D, Srivastava, HM, Yang, XJ: Local fractional variational iteration algorithms for the parabolic Fokker-Planck equation defined on Cantor sets. Prog. Fract. Differ. Appl. 1, 1-11 (2015)

44. Saravanan, A, Magesh, N: An efficient computational technique for solving the Fokker-Planck equation with space and time fractional derivatives. J. King Saud Univ., Sci. 28, 160-166 (2016)

45. Deng, WH: Finite element method for the space and time fractional Fokker-Planck equation. SIAM J. Numer. Anal. 47, 204-226 (2008)

46. Deng, KY, Deng, WH: Finite difference/predictor-corrector approximations for the space and time fractional Fokker-Planck equation. Appl. Math. Lett. 25, 1815-1821 (2012)

47. Kullberg, A, del-Castillo-Negrete, D: Transport in the spatially tempered, fractional Fokker-Planck equation. J. Phys. A, Math. Theor. 45, 255101 (2012)

48. Kullberg, A, del-Castillo-Negrete, D: Levy ratchets in the spatially tempered fractional Fokker-Planck equation. Physics 45, 5826-5831 (2010)

49. Gajda, J: Fractional Fokker-Planck equation with space dependent drift and diffusion: the case of tempered $\alpha$-stable waiting-times. Acta Phys. Pol. 44, 1149-1161 (2013)

50. Chen, MH, Deng, WH: Discretized fractional substantial calculus. ESAIM Math. Model. Numer. Anal. 49, 373-394 (2015)

51. Guo, BL, Pu, XK, Huang, FH: Numerical Solution of Fractional Partial Differential Equations. Science Press, Beijing (2011)

52. Podlubny, I: Fractional Differential Equations. Academic Press, New York (1999)

53. Li, CP, Zeng, FH: Numerical Methods for Fractional Calculus. CRC Press, Boca Raton (2015)

54. Chen, MH, Deng, WH, Barkai, E: Numerical algorithms for the forward and backward fractional Feynman-Kac equations. J. Sci. Comput. 62, 718-746 (2015) 
55. Li, C, Deng, WH, Wu, YJ: Numerical analysis and physical simulations for the time fractional radial diffusion equation. Comput. Math. Appl. 62, 1024-1037 (2011)

56. Henry, BI, Langlands, TAM, Wearne, SL: Anomalous diffusion with linear reaction dynamics: from continuous time random walks to fractional reaction-diffusion equations. Phys. Rev. E 74, 031116 (2006)

57. Langlands, TA, Henry, BI, Wearne, SL: Anomalous subdiffusion with multispecies linear reaction dynamics. Phys. Rev. E $77,021111(2008)$

Submit your manuscript to a SpringerOpen ${ }^{\circ}$ journal and benefit from:

- Convenient online submission

$\checkmark$ Rigorous peer review

Open access: articles freely available online

- High visibility within the field

- Retaining the copyright to your article

Submit your next manuscript at $\gg$ springeropen.com 